DOI: https://doi.org/10.46296/yc.v4i7edesp.0065

\title{
ESTRATEGIAS COMPETITIVAS DE LOS PRESTADORES DE SERVICIOS DE ALOJAMIENTO DEL SECTOR TURÍSTICO Y SU SOSTENIBILIDAD EN LA PARROQUIA CRUCITA DEL CANTÓN PORTOVIEJO PROVINCIA DE MANABÍ. \\ COMPETITIVE STRATEGIES OF ACCOMMODATION SERVICE PROVIDERS IN THE TOURISM SECTOR AND THEIR SUSTAINABILITY IN THE CRUCITA PARISH OF THE PORTOVIEJO CANTON OF MANABÍ PROVINCE.
}

\author{
Muñoz-Briones Jorge Eduardo ${ }^{1 *}$; Tamayo-Cevallos Christian David ${ }^{2}$ \\ ${ }^{1}$ Estudiante de la Maestría en Administración de Empresas / Instituto de Posgrado / \\ Universidad Técnica de Manabí, UTM. Portoviejo, Ecuador. \\ ${ }^{2}$ Docente Titular Facultad de Ciencias Administrativas y Económicas / Universidad \\ Técnica de Manabí, UTM. Portoviejo, Ecuador. \\ *Correo: jorge_munozb@hotmail.com
}

\begin{abstract}
Resumen
El presente estudio tiene como objetivo determinar las estrategias competitivas aplicables por los alojamientos turísticos de la parroquia Crucita en un sector en decadencia. La metodología fue de tipo descriptiva y la recolección de la información se realizó a través de la revisión de los datos estadísticos del catastro de Manabí del Servicio de Rentas Internas, donde se pudo constatar que la parroquia Crucita posee 33 registros de alojamientos, 15 de estos registros han permanecido en el sector por más de 15 años. Con base a ello, la población de estudio está constituida por los propietarios y/o administradores que laboran en estos 15 registros de alojamientos. Mediante la técnica de la encuesta y la aplicación del instrumento, se obtuvo la información y datos que permitieron conocer las causas del problema de investigación. El instrumento fue una entrevista estructurada de preguntas mixtas y aquellas de opción cerrada fue dicotómica permitiendo determinar el entorno competitivo del sector turístico de Crucita. Los resultados permitieron indicar que la situación de la demanda y de barreras de salidas, fueron considerados como un entorno inhóspito y que el factor de rivalidad en un $71 \%$ de las opiniones se debe a clientes débiles y fragmentados.
\end{abstract}

Palabras clave: estrategias competitivas, alojamientos turísticos, Crucita.

\begin{abstract}
The present study aims to determine the competitive strategies applicable to tourist accommodation in the Crucita parish in a sector in decline. The methodology was descriptive, and the information was collected through the review of statistical data from the Manabí catastro of the Internal Revenue Service, where it was found that the Crucita parish has 33 accommodation records, 15 of these records have remained in the sector for over 15 years. Based on this, the study population is made up of the owners and / or administrators who work in these 15 accommodation records. By means of the survey technique and the application of the instrument, the information and data that allowed to know the causes of the research problem were obtained. The instrument was a structured interview of mixed questions, and those with a closed option were dichotomous, allowing the competitive environment of the Crucita tourism sector to be determined. The results indicated that the situation of demand and exit barriers were considered an inhospitable environment and that the rivalry factor in $71 \%$ of opinions is due to weak and fragmented customers.
\end{abstract}

Keywords: competitive strategies, tourist accommodation, Crucita.

Información del manuscrito:

Fecha de recepción: 15 de septiembre de 2020.

Fecha de aceptación: 12 de noviembre de 2020.

Fecha de publicación: 16 de noviembre de 2020. 


\section{Introducción}

El turismo como política de Estado y como sector estratégico en Ecuador busca diversificar la economía y por tanto mejorar la calidad de vida de los habitantes. En la actualidad han existido iniciativas legislativas a favor de la activación de este sector estratégico, con lo cual se le está dando al turismo una merecida prioridad y, por tanto, se incrementa la posibilidad de que genere rentabilidad para el país y oportunidades de empleo. La literatura menciona que la implementación del Plandetour 2020 que desarrolló el Ministerio de Turismo permitirá posicionar al Ecuador como un destino turístico líder altamente diverso, competitivo, seguro y de calidad en el ámbito internacional; así garantizando el desarrollo y operación turística de sus riquezas culturales y naturales y dentro de ellas el desarrollo de los sectores turísticos como es el caso del cantón Portoviejo (Consejo Parroquial de Gobierno, 2010-2014).

Estos objetivos pueden ser transferidos a cada uno de los cantones y las parroquias con el objeto de impulsar las actividades económicas de sector turismo y por tanto impulsar el desarrollo de iniciativas que creen oportunidades de encadenamiento productivo en la zona y con ello mejorar las condiciones de vida de los habitantes, particularmente en las zonas rurales con potencialidades para el turismo, tal como sucede en el Cantón Portoviejo.

Para ello se necesita que se lleven a cabo estrategias competitivas destinadas a los prestadores de servicios de alojamiento del sector turístico en búsqueda de alcanzar la sostenibilidad en la parroquia Crucita del cantón Portoviejo provincia de Manabí. Las estrategias competitivas de los prestadores de servicios de alojamiento o también llamados hoteles, hostales $y$ hosterías de la parroquia de Crucita, los cuales son de gran importancia para el cantón, toda vez que, de ser ejecutadas de manera correcta, suponen una dinamización de la economía local a través del turismo interno y la visita de turistas extranjeros.

En relación a lo planteado y contextualizando específicamente la situación presente en la comunidad 
de Crucita, se tiene que en los últimos 10 años esta zona ha alcanzado un desarrollo notable por el potencial de sus recursos naturales, siendo la pesca artesanal e industrial, que, sumadas a la actividad de la agricultura, representan la ocupación de más del $80 \%$ de la población, siendo además la población de la parroquia Crucita considerada en su totalidad como Rural

Es importante considerar que la principal actividad económica en Crucita según el Consejo Parroquial de Gobierno (2010-2014) es la agricultura con un $55 \%$, la pesca es sólo es del $21.5 \%$. El comercio con el $10 \%$ y el turismo tan solo un $5.5 \%$, siendo la actividad que debería generar mayor riquezas y fuentes de trabajo por ser una Parroquia beneficiada por sus múltiples atributos naturales; y otras actividades con un $5.7 \%$, tales como: la producción de harina de pescado, actividad camaronera y fábrica de hielo; sin embargo, en los últimos años ha aumentado el interés de nacionales y extranjeros en la construcción de un número de casas modernas y hoteles en esta comunidad.
En este sentido, la falta de inversión pública ha limitado su desarrollo y no ha logrado el sitial que le correspondería por problemas de índole político-administrativo; así pues, no cuenta con infraestructura básica sanitaria que es una limitante para la implementación de sitios de alojamiento y de recreación. En otro sentido, también influye el recurso humano que no está debidamente capacitado para la prestación de estos servicios, aspectos que de alguna manera tiene incidencia en el ambiente social, económico, cultural, natural, entre otros (ecotec.edu.Ecuador, s.f.).

Por otra parte, la mayoría de los alojamientos turísticos tienen poca capacidad instalada y el alquiler de las habitaciones ha permanecido bastante bajo, promediando un costo de $\$ 20,00$ por habitación y $\$ 10,00$ por persona, en hoteles más cómodos hasta $\$ 40,00$ por habitación y \$20,00 por persona (Consejo provincial de Portoviejo., 2010), con lo cual el ingreso por este concepto ha venido disminuyendo. En la actualidad, sector hotelero de Crucita está compuesto por un total de 29 hoteles, según el censo realizado por la Asociación de 
Empresarios Hoteleros de Crucita en 2019 (Márquez, Cuétara, Bernándo, \& Vera, 2020).

Por cuestiones de estrategia, sería indispensable y como una alternativa, descentralizar la estructura de alojamiento en el centro de Crucita, para disminuir la saturación del atractivo y equilibrar la capacidad de carga de los destinos turísticos con un mínimo de impacto (Consejo Parroquial de Gobierno, 2010-2014)

Partiendo de lo anterior, resulta necesario aplicar una herramienta que permita determinar la fuerza competitiva del sector turístico y su caída a nivel local, en consecución de las estrategias que pueden aplicar los alojamientos turísticos de Crucita ya sea para la permanencia - salida del sector con el menor impacto posible a la organización.

Por consiguiente, a través de la identificación de los alojamientos y su apreciación respecto a la fuerza de la competencia del sector en decadencia, permitirá determinar el comportamiento de la demanda, las barreras de salida del sector y nivel de competencia, dando un panorama general de las estrategias a aplicar de manera individual y por sector.

Una vez realizado el análisis pertinente, desde la perspectiva de los propietarios y administradores de los alojamientos de la parroquia Crucita consideran al sector como inhóspito en un $53 \%$ y un $47 \%$ lo considera hospitalario respecto a la intensidad de la competencia. Así mismo, un $29 \%$ considera que existe una posición fuerte de las bolsas de la demanda subsistente, mientras que un $71 \%$ considera que existe una posición débil de las bolsas de demanda subsistentes.

De esta manera, el $14 \%$ de los alojamientos podrían tomar estrategias de primicia y/o cosecha, el $15 \%$ podrían tomar estrategias de hueco y/o cosecha, el 34 \% podrían tomar estrategias de desinversión rápida y/o cosecha, y por último el $38 \%$ podrían considerar una estrategia de desinversión rápida.

\section{Metodología}

El proceso metodológico se desarrolló bajo el paradigma cuantitativo se representarán información estadística los cuales representan la realidad sobre el 
sector turístico en el sector de Crucita. Del mismo modo se desarrolla bajo el método hipotético deductivo el cual permite realizar generalizaciones a partir de una hipótesis previa.

Por otra parte, para llevar a cabo el procedimiento de la investigación se desarrolló en un primer momento procesos de revisión documental para enunciar las teorías que sustentan el estudio de los fenómenos y procesos y en un segundo momento, procesos propios de la investigación de campo, proceso que la técnica de campo permite la observación en contacto directo con el objeto de estudio, y el acopio de testimonios que permitan confrontar la teoría con la práctica en la búsqueda de la verdad objetiva (Bernal Torres, 2006).

Estos procesos se detallan:

Levantamiento del Catastro de los establecimientos turísticos de Crucita, basados en el catastro del Servicio de Rentas Internas de Manabí en la que está realizado un inventario de las empresas turísticas de Crucita, es decir el número total de empresas de alojamiento. Del mismo modo en este registro se evidencia aquellos que estén en restauración y servicios complementarios, la categoría de cada uno de ellos. Además de reflejarse los propietarios de las empresas y sus datos. Esta información permite realizar un estudio de los servicios que presta actualmente la localidad y así determinar la calidad de estos.

Se establecieron los criterios para la selección de la muestra a estudiar atendiendo a los años de servicio.

Se utilizó la herramienta FODA, con esta herramienta se podrá analizar las fortalezas de las empresas; humanas, económicas, sociales, normativas etc. Las oportunidades que ofrece el contexto institucional, social y así mismo sus amenazas y por último sus debilidades aplicadas a la situación empresarial turística de Crucita, lo cual ayudará a que el análisis se realice objetivamente.

Las 5 Fuerzas de Porter es otra herramienta que se utilizará en el análisis de estrategias del final de partida para los sectores en declive.

En resumen, las herramientas utilizadas para la evaluación de la 
gestión de las empresas turísticas ayudarán para saber qué está pasando con la empresa, hacia dónde se quiere llegar, cómo se quiere llegar y qué se está haciendo para lograr sus objetivos

\subsection{Tipo de investigación}

La presente investigación es de tipo descriptiva, dado que se pretendió caracterizar cada una de las variables en estudio, tal como se presentan en la realidad estudiada (Bernal Torre, 2010, pág. 202). Además de ser de tipo aplicada, tomando en cuenta que se pretende generar una solución a la realidad encontrada con el fin de proponer estrategias competitivas que privan en los alojamientos del sector turístico de crucita en decadencia.

\subsection{Población objeto de estudio}

Posterior a la revisión de los datos estadísticos del catastro de Manabí del Servicio de Rentas Internas, se pudo constatar que la parroquia Crucita posee 33 registros de alojamientos. Con base a ello, se realizó un muestreo intencionado, teniendo como criterios para la selección de la muestra:
Permanencia de la empresa turística en el sector mayor a 15 años

\subsection{Empresas activas para el momento}

Partiendo de estos criterios se encontraron 15 registros de alojamientos revisados en el catastro del SRI, sin embargo, en este registro constaban algunos nombres repetidos. En tal sentido, una vez depurados los registros se identificaron 9 y de estos registros 2 ya no estaban en funcionamiento; por lo que la muestra quedó conformada por un total de 7 alojamientos y por los propietarios y/o administradores que laboran en estos.

\subsection{Técnicas de recolección de la información}

Para la recolección de la información se empleó la técnica de la encuesta y como instrumento una entrevista estructurada, con el objeto de conocer las características del sector hotelero en Crucita, específicamente lo relativo a los factores que pudiesen intervenir en la decadencia de la industria. En relación con el instrumento, las preguntas fueron tipo mixtas, teniendo como opciones 
de respuesta la SI y No y con ello determinar el entorno competitivo del sector turístico de Crucita

\section{Resultados}

\subsection{Situación de la demanda}

De la revisión de la situación de la demanda, de los datos recolectados, se obtiene que existe total conciencia, por parte de los propietarios y/o administradores de alojamientos de que el sector está en descenso y por tanto, se encuentran dentro de un entorno hospitalario. Por otro lado, el $70 \%$ considera que no existen bolsas de demandas e importas y el $57 \%$ indicó que no existe fidelidad por parte de la clientela, y el 86 \% señaló que no se pueden fijar precios altos; colocando la situación de la demanda en un entorno inhóspito.

Del presente análisis de la demanda se determina que se considera que el entorno es inhóspito en un $54 \%$, y que existe una débil posición de la demanda en las bolsas existentes.

\subsection{Barreras de salida}

Como resultado de las encuestas para valorar las barreras de salida, se tiene que el $57 \%$ considera que se puede mantener sin hacer inversiones necesarias, que el $57 \%$ considera que tiene poca capacidad de alojamiento, que el $71 \%$ considera que el bien inmueble es viejo y que el $71 \%$ considera tener una débil integración vertical, ponderaciones que enmarcan al entorno del sector como hospitalario. Por otro lado, el $86 \%$ considera que no es fácil vender el bien inmueble, que el $57 \%$ considera que tiene instalaciones compartidas y un 100 $\%$ considera que existen muchos competidores; ponderaciones que enmarcan al entorno del sector como inhóspito.

De esta manera con un $55 \%$ en entrono para las barreras de salida es considerado por el sector como inhóspito, y el $45 \%$ considera que es hospitalario.

\subsection{Factores de rivalidad}

Del análisis del factor de rivalidad, se determina que el $71 \%$ considera que los clientes son débiles y segmentados en marcando al entorno como hospitalario. Por otra parte, el $29 \%$ considera que el costo de buscar un nuevo cliente es alto, colocando al entorno como inhóspito. Considerando lo 
expuesto, de la ponderación realizada se desprende que para el factor de rivalidad el entorno es hospitalario.

\subsection{Aplicación de la Matriz de estrategias para la fase de decadencia}

Del análisis del factor de rivalidad, se determina que el $71 \%$ considera que los clientes son débiles y segmentados en marcando al entorno como hospitalario. Por otra parte, el $29 \%$ considera que el costo de buscar un nuevo cliente es alto, colocando al entorno como inhóspito. Considerando lo expuesto, de la ponderación realizada se desprende que para el factor de rivalidad el entorno es hospitalario.

Figura 1. Matriz de estrategias para la fase de decadencia.

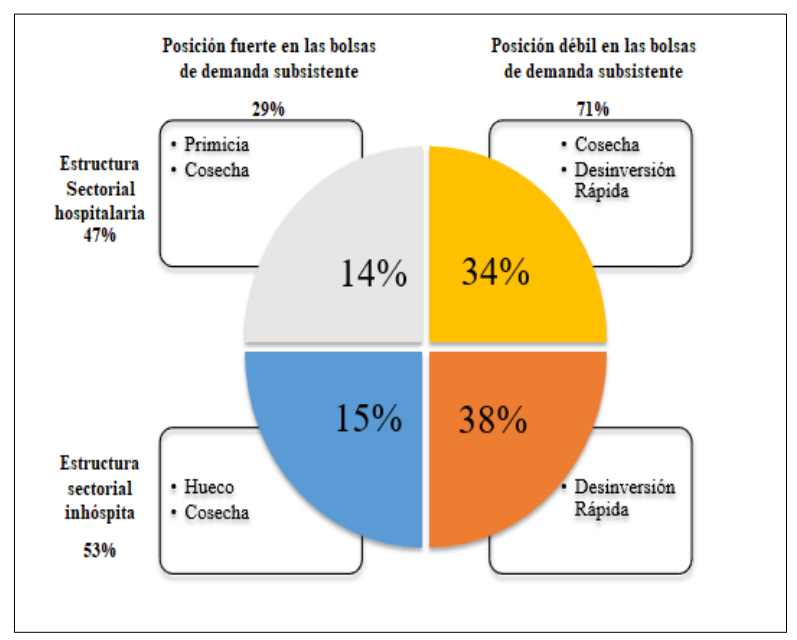

Elaborado por: Autores de la investigación Fuente: Adaptación de (Porter Michael, 2003, p. 120).

\section{Conclusiones}

En el marco de los factores estructurales de los alojamientos turísticos de la parroquia abordados en el presente trabajo, logran expresarse los principales resultados, alcances y proyecciones Crucita y atendiendo los objetivos propuestos, se presentan las siguientes conclusiones:

Según la apreciación de los propietarios de alojamientos y administradores encuestados con respecto a la situación de la demanda en un $54 \%$ consideró inhóspito al sector, considerando que la caída de la demanda es muy lenta y predecible, que no existen varias e importantes bolsas de demanda con fidelidad a la marca lo cual imposibilita colocar precios altos.

En cuanto a las barreras de salida el 55 \% consideró inhóspito al sector, toda vez que se tienen que realizar reinversiones grandes y frecuentes, donde el exceso de capacidad es sustancial y muy difícil vender el bien inmueble, también el costo de desmantelamiento es alto y existen mucho competidores. Por otra parte, el $45 \%$ considera al sector como 
hospitalario, detalle que corresponde a que los alojamientos en su mayoría son relativamente pequeños en cuanto al bien inmueble, lo contrario pasa con aquellos bienes inmuebles que por su tamaño resultan difíciles de vender, tales como la hostería de segunda categoría como el hotel de tercera categorías, los cuales además de tener las mayor capacidad alojamiento, tienen la mayor cantidad de bienes muebles de los cuales también resulta difícil vender.

Los factores de rivalidad en un $71 \%$ señalaron que los clientes son débiles y fragmentados lo que hace el entorno hospitalario, esto se corresponde a aquellos alojamientos que han encontrado ciertos tipos de demandas que les permiten permanecer en el sector, clientes que aceptan y busca el bien y el servicio prestado por el establecimiento. Esto pude deberse a la aplicación de nuevas tecnologías para el contacto con los clientes o prestaciones de servicios diferenciados que requieren inversión que consideran alto.

\section{Bibliografía}

Álava Macías, M. I. (2018). El turismo en la reactivación económica de la provincia de Manabí. Tesis, 99. Guayaquil, Guayas, Ecuador: Universidad Católica Santiago de Guayaquil.

Arroyo Cobeña, M. (2018). Importancia del turismo para el desarrollo local de la provincia Manabí. 3, 5. doi:2528-8075

Bernal Torre, C. (2010). Metodología de la investigación (Tercera edición ed.). Colombia: PEARSON EDUCACIÓN. doi:978-958-699-128-5

Bilbao, P. (2013). Política de Empresa y Estrategia. Editorial Universitas, $3^{a} \mathrm{Ed}$.

Consejo Parroquial de Gobierno . (2010-2014). Plan Estratégico De Desarrollo Turístico 20102014 Parroquia Crucita Del Cantón Portoviejo, Provincia De Manabí.

Consejo provincial de Portoviejo. (2010). Plan estratégico de desarrollo turístico de Crucita 2010-2014.

Constitución de la República del Ecuador, Registro Oficial 449 de 20-oct.-2008 (2008). Obtenido de https://www.seps.gob.ec/docu ments/20181/25522/CONSTI TUCION\%20DE\%20LA\%20R 
EPUBLICA\%20DEL\%20ECU ADOR\%20actualizada\%20ab ril\%202019.pdf/e228157e9cd5-446e-bbdf152 cea50cbd9

Contreras Sierra, E. (2013). EI concepto de estrategia como fundamento de la planeación estratégica. Pensamiento y Gestión, 30. doi:ISSN 16576276

Court Monteverde, E., \& Williams Rengifo, E. (2011). Estadísticas y Econometría Financeiro. Buenos Aires: Cengage Learning Argentina. doi:978-987-1486-86-1

Cuadrado Roura, J., \& López Morales, J. (2011). El turismo: Un sector clave en la economía española. 35 .

David, F. (2003). Conceptos de Administración Estratégica. Mexico: PEARSON EDUCACIÓN. doi:ISBN 97026-0427-3

Ecotec.edu.Ecuador. (s.f.). CAPÍTULO II-MERCADEO Y COMERCIALIZACIÓN.

Obtenido de https://www.ecotec.edu.ec/do cumentacion/investigaciones/ estudiantes/trabajos_de_grad o/544_2010_MKT_CEDE\%C 3\%91OG_2.pdf

L. Hill, C., \& Jones, G. (2009). Adminstración Estratégica (Octava edición ed.). Mexico:
McGRAW-HILL. doi:978-97010-7269-1

Ley de Turismo. (2008). Ley de Turismo. Obtenido de https://www.asambleanaciona I.gob.ec/es: Registro Oficial Suplemento 733 de 27-dic2002

Loor, L., Alonso, A., \& Pérez, M. (2018). La Actividad Turistica en el Ecuador: ¿Turismo Consciente 0 Turismo Tradicional? Revista ECA Sinergia. Facultad de Ciencias Administrativas y Económicas. U.T.M.vOL. 9. Núm.1.

Márquez, L., Cuétara, L., Bernándo , J., \& Vera , D. (2020). Sistema de indicadores para la evaluación de la sostenibilidad económica del sector hotelero en la parroquia Crucita, Manabí, Ecuador. 41 (3), Pág. 3.

MINTUR. (2002). Reglamento General De Actividades Turísticas, Reglamento General De Actividades Turísticas (Decreto No. 340. Obtenido de https://www.hotelesecuador.c om.ec/downloads/Reglament 0\%20General\%20de\%20Acti vidades\%20Turisticas.pdf

Mogro, S., \& Guale Córdova, A. (2017). Estudios Sectoriales: Alojamiento y Servicio de Comidas. Obtenido de http://portal.supercias.gob.ec/ 
wps/wcm/connect/a0a93fff-

5441-45aa-807a-

$8 \mathrm{ff} 010875 \mathrm{c} 4 \mathrm{~d} / \mathrm{estudio}+\mathrm{de}+\mathrm{alo}$

jamiento $+y+$ servicios $+\mathrm{de}+\mathrm{co}$

mida.pdf?MOD=AJPERES\&C

ACHEID=a0a93fff-5441-

45aa-807a-8ff010875c4d

Plan Estratégico De Desarrollo

Turístico Del Cantón

Portoviejo 2017 - 2022.

(2017). Plan Estratégico De

Desarrollo Turístico Del

Cantón Portoviejo 2017 2022. FIMCBOR - TURISMO 2017.

Plandetur 2020. (2007). Diseño Del Plan Estratégico De Desarrollo De Turismo Sostenible Para Ecuador "Plandetur 2020". Banco Interamericano de Desarrollo. Cooperación Técnica no ATN/FG-9903-EC. Obtenido de https://www.turismo.gob.ec/w pcontent/uploads/2015/04/proy ecto_plandetur_2011_2014V ERSION2.pdf

Porter, M. (2003). Ser competitivo. Barcelona: Ediciones Deusto. doi:84-234-2113-9

Reglamento de Alojamiento Turístico. (2016). Reglamento de Alojamiento Turístico. Obtenido de https://www.asambleanaciona I.gob.ec/es: Registro Oficial Suplemento 465 de 24-mar.2015
Schroeder, R. G., Meyer Goldstein, S., \& Rungtusanatham, M. (2005). Administración de Operaciones. Conceptos y casos contemporáneos (Quinta ed.). MEXICO: McGRAW-HILL. Obtenido de www.elosopanda.com 